
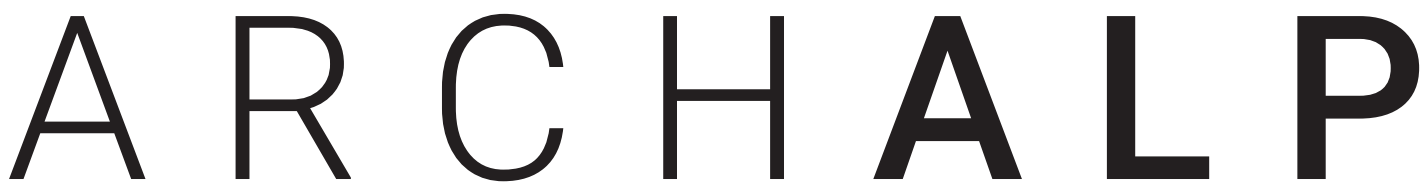

Rivista internazionale di architettura e paesaggio alpino / Revue internationale d'architecture et de paysage dans les Alpes / Internationale Zeitschrift für Alpine Architektur und Landschaft / Revija za alpsko arhitekturo in pokrajino / International journal of alpine architecture and landscape

Rer una nuota abitabilita delle Alpi.

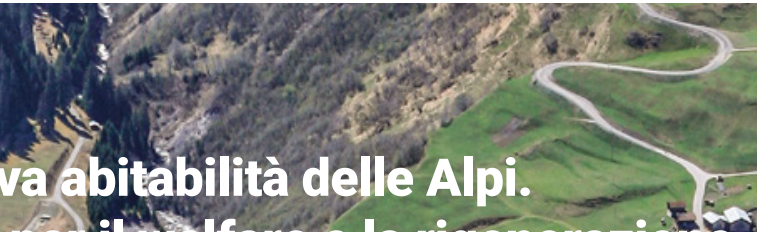
Architeture pet ilf lfare e la rigenerazione For a new inhabifability of the Alps. Architectures for welfare ând regeneration $/$ Pour une nouvelle habitabilité des Alpes. Architectures pour le welfare et la régéhérationy Für eine núue Bewohnbarkeit in den Alpen. Architekturen für Wohf ahrt und Regeneration / Za novo. bivalnost v Alpah, arhitekture za dobrobit in regeneracijo
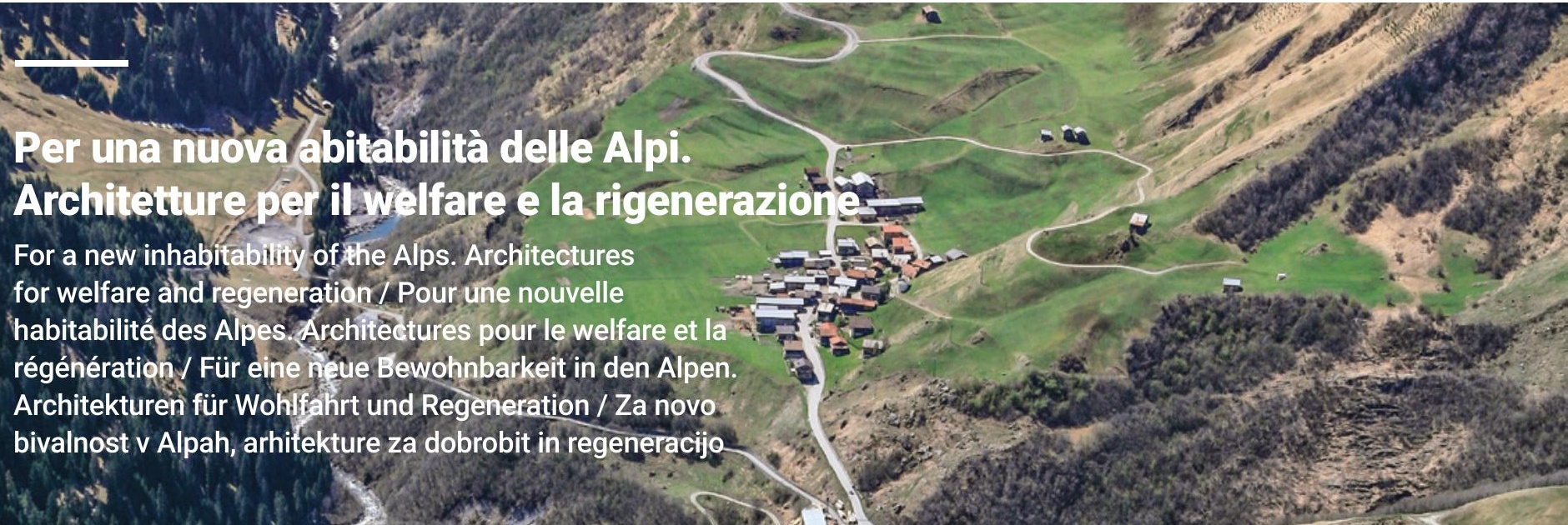
a $x \rightarrow 3$
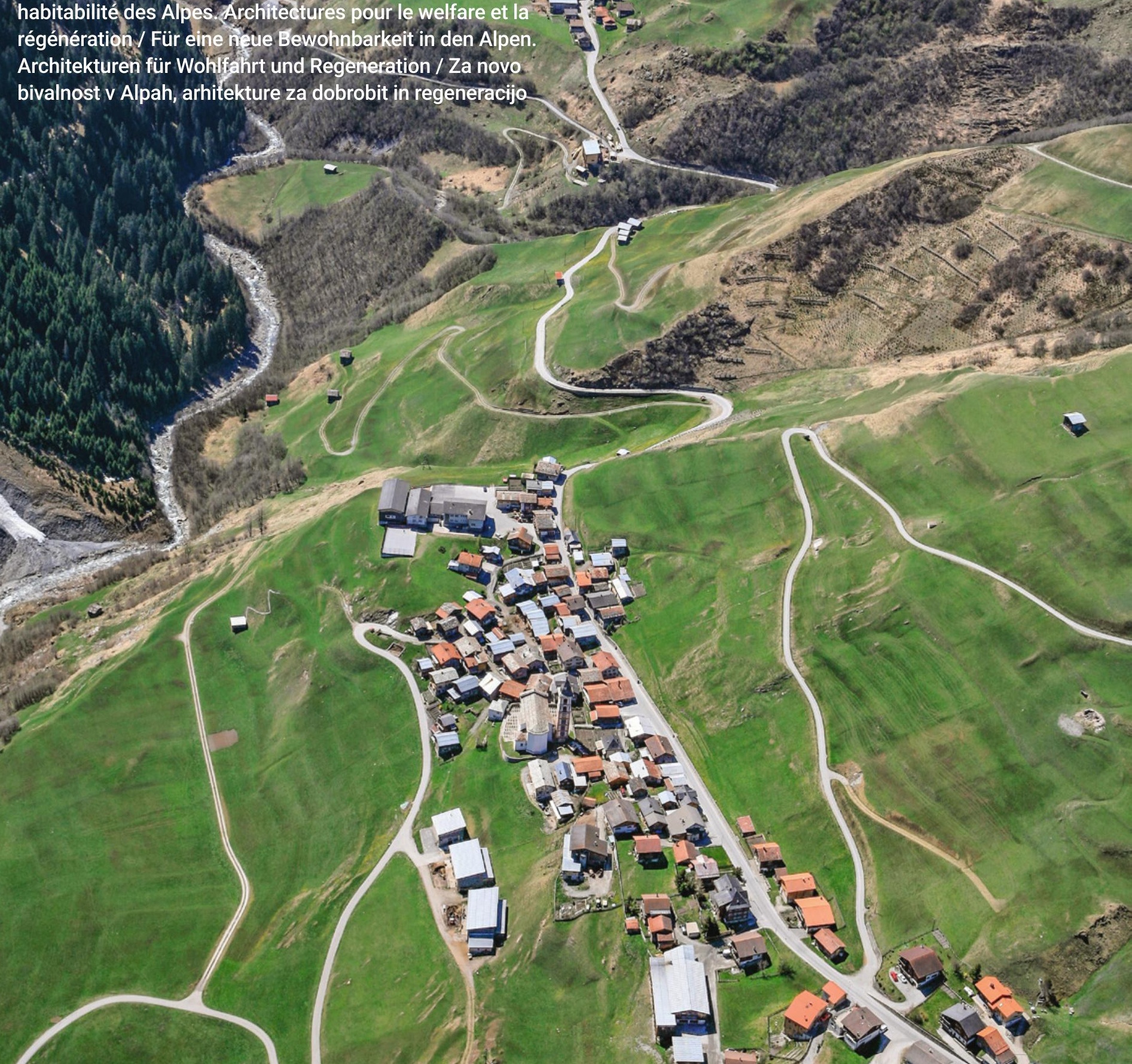


\section{Indice dei contenuti Contents}

\section{Temi}

Sulla centralità di spazio e territorio nel progetto di rigenerazione delle montagne e delle aree interne / On the centrality of space and territory in the project of regeneration of mountains and internal areas Antonio De Rossi, Laura Mascino

I servizi nelle Alpi italiane: quali e dove? Idee per uno scenario post-pandemico / Facilities in the Italian Alps: which ones and where? Ideas for a post-pandemic scenario

Giuseppe Dematteis

Manifesto di Camaldoli per una nuova centralità della montagna

Alla ricerca della distanza perduta. Rigenerare luoghi, persone e immaginari del riabitare alpino / In search of the lost distance. Regenerating places, people and images related to Alpine reinhabitation

Filippo Barbera, Andrea Membretti

Futuro e rigenerazione

Marco Bussone

\section{Esperienze}

Dorferneuerung zwischen Erhalten und Gestalten / 
Valades ousitanes, architettura e rigenerazione / Valades ousitanes,

Ostana e Topolò: hardware, software e welfare nelle comunità di "ritorno" / Ostana and Topolò: hardware, software and welfare in "return" communities

Margherita Valcanover

"Senza mostrare i muscoli": i progetti di rigenerazione di Tao+C e AZL nel solco della "prosperosa società" della Cina contemporanea / "Without flexing one's muscles": the regeneration projects of Tao+C and AZL in the wake of the "prosperous society" of contemporary China Edoardo Bruno, Dalila Tondo

L'archipel Butor. Une régénération, par la culture, d'un village soumis à la métropolisation genevoise / The Butor archipelago. A regeneration, through culture, of a village subject to the metropolisation of Geneva Arnaud Dutheil

Pratiche e progettualità di rigenerazione e welfare: il "Premio triennale Giulio Andreolli - Fare paesaggio" / Actions and projects of regeneration and welfare: the "Premio triennale Giulio Andreolli - Fare paesaggio" Giorgio Tecilla

Architetture e strategie per il welfare. Il caso di Brunico in Val Pusteria / Welfare architectures and strategies. The Bruneck case in Val Pusteria

Eleonora Gabbarini

Arhitektura oživlja / Architecture revives Kristina Dešman, Maja Ivanič

Si Crans-Montana meurt. Soigner le corps malade d'une station / If Crans-Montana dies. Taking care of the ailing body of a tourist resort Patrick Giromini 


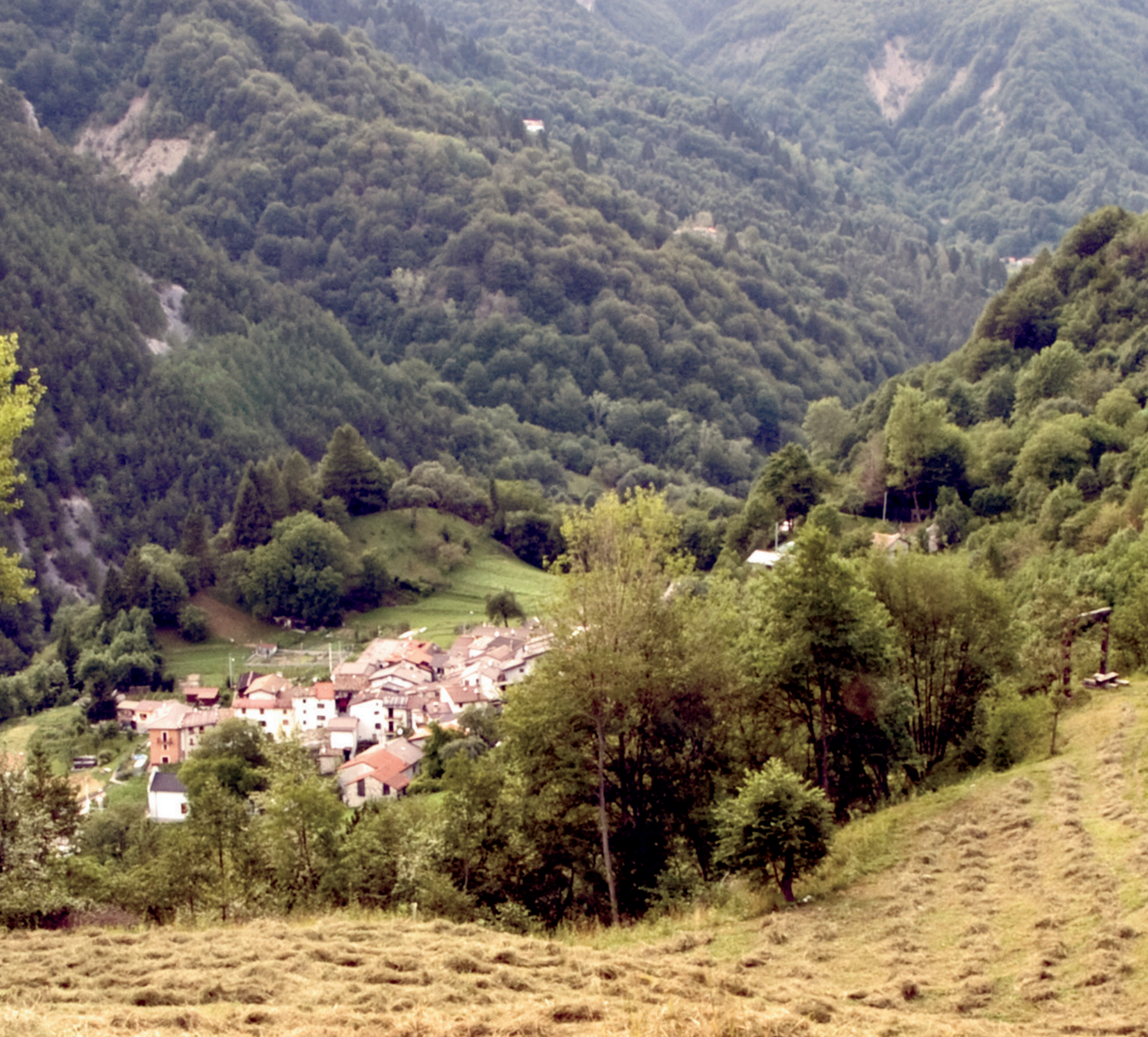

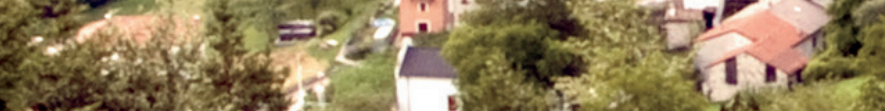

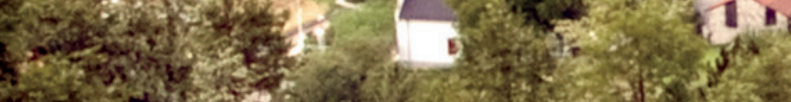

(3)

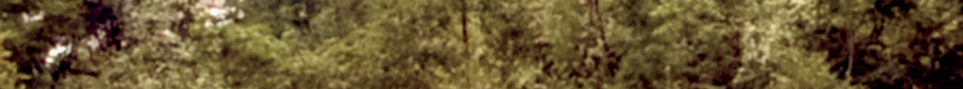

ats

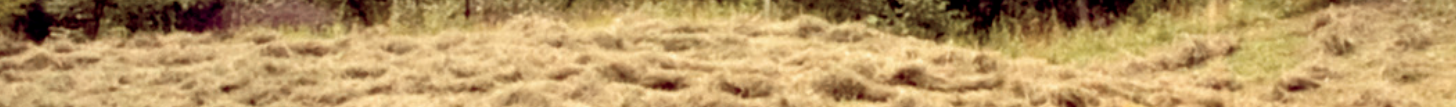

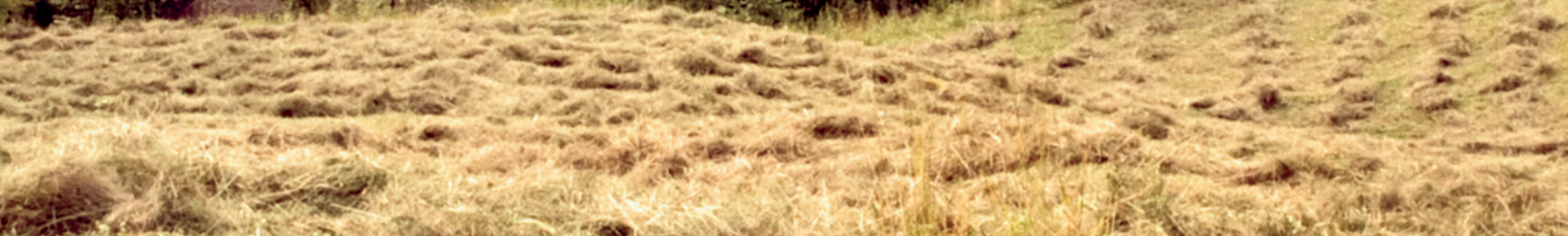

W.

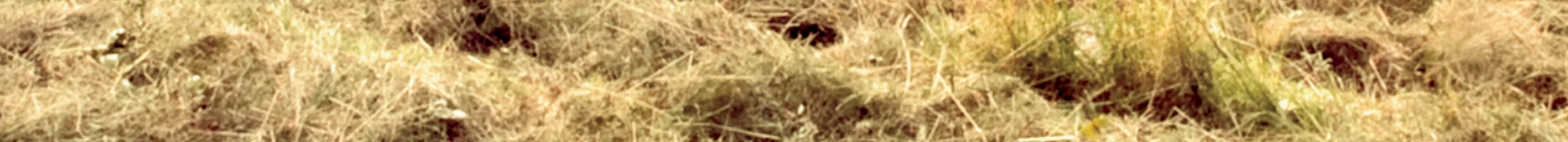

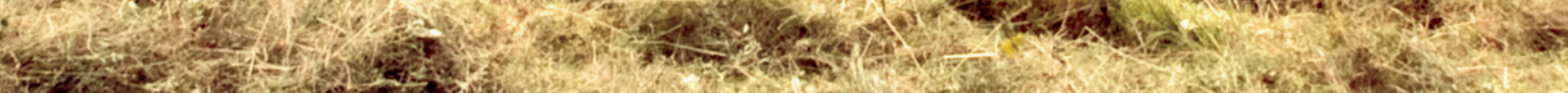

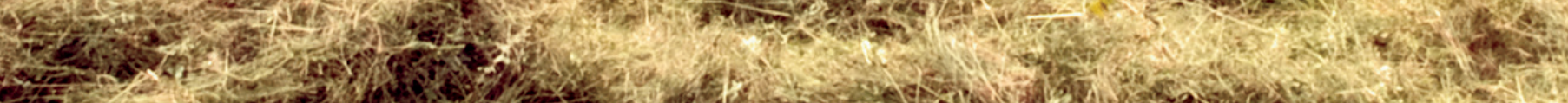

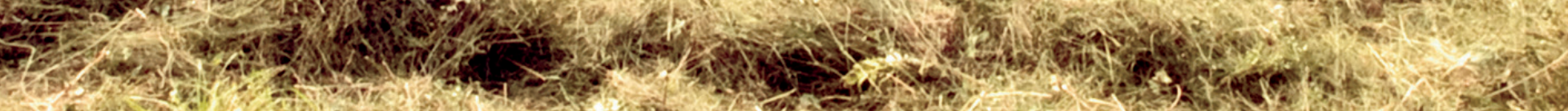

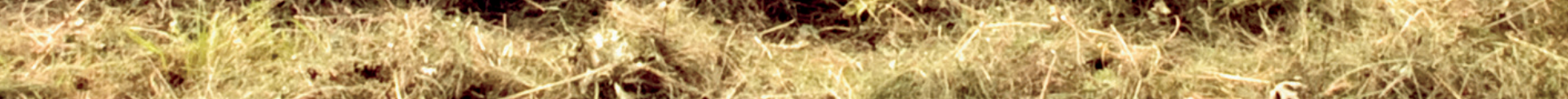

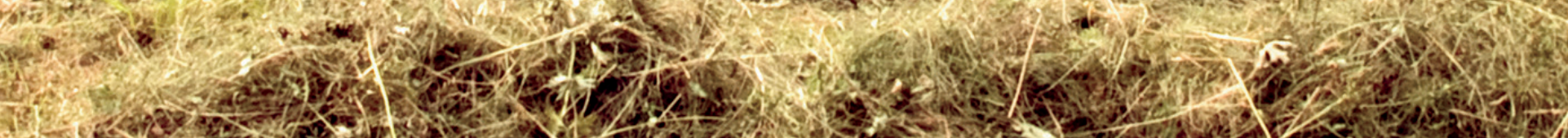

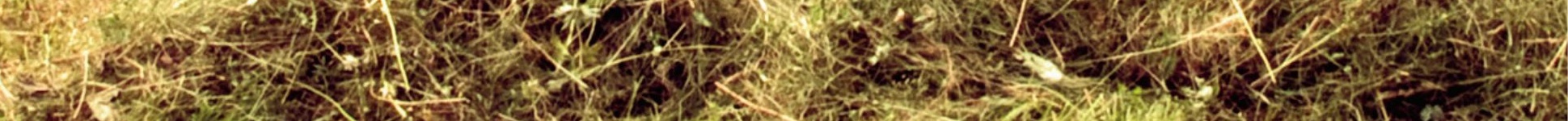

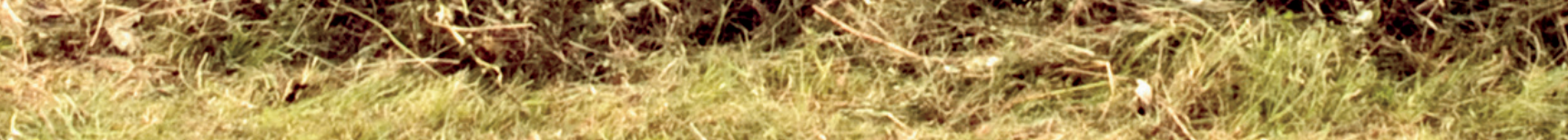

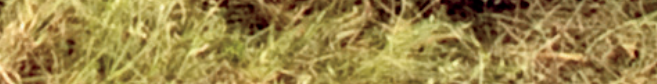
3 ese

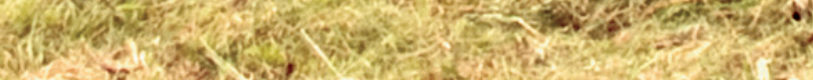

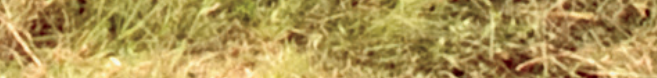




\title{
Alla ricerca della distanza perduta. Rigenerare luoghi, persone e immaginari del riabitare alpino
}

In search of the lost distance.

Regenerating places, people and images of Alpine reinhabitation

\begin{abstract}
Living in a territory means being able to take part in the "functioning of citizenship" related to moving, feeding, saving, buying, participating, taking care, thinking about the future, investing in life plans, assuming social and political responsibility. Living in a territory is the way in which rights related to the status of "resident" become ways of "being or doing" that constitute the well-being of people as citizens. Citizenship is a daily opportunity, dependent on the characteristics of the context. Irreducibly different from the romantic images that described it as isolated, remote, physically separated from the modern world of cities and social change, in the last seven centuries the Alps have built a complex civilization around the multidimensional axis of the "right distance". Today, in the face of the COVID-19 pandemic, such "right distance" can be an antidote to the disorganized and individualistic escape of the wealthy classes who can afford it. Re-inhabiting the marginalized places becomes instead the result of a political reversal of the gaze, of a radical and collective change of perspective.
\end{abstract}

\section{Filippo Barbera}

Professor of Economic Sociology at the Department of Culture, Politics and Society of the University of Turin and affiliated with the Collegio Carlo Alberto. His research interests are in the areas of social innovation, foundational economy and development of marginal areas. His recent publications include Alternative Food Networks: An Interdisciplinary Assessment, 2018, Palgrave Macmillan (edited with A. Corsi, E. Dansero, C. Peano), Foundational Economy, Manchester University Press, 2018 (as "Collective for the foundational economy") and The Foundational Economy and Citizenship (edited with I.R. Jones, Bristol, Policy Press 2020).

\section{Andrea Membretti}

PhD in Sociology and Senior Researcher at EURAC (Bolzano), where he is the Scientific coordinator of the Horizon 2020 Project MATILDE. He is also Associate Professor of Sociology at the University of the Free State (South Africa) and Assistant Professor of Sociology of the Territory at the University of Pavia. His main field of study is migration to European and non-European mountain and rural territories.

Keywords

Alps, regeneration, reinhabitation, social innovation, citizenship. 


\section{Come (ri)abitare un territorio?}

Abitare un territorio è molto più che avere la residenza (o il domicilio) in quel luogo. Abitare non è un codice di avviamento postale. Abitare significa essere in grado di assumere $i$ "funzionamenti di cittadinanza" legati al muoversi, nutrirsi, risparmiare, acquistare, partecipare, curare, pensarsi nel tempo, investire in piani di vita, assumere responsabilità sociale e politica. Abitare un territorio è il modo attraverso cui i diritti legati allo status di "residente" diventano stati (modi) di "essere o di fare" che costruiscono il benessere delle persone come cittadini. In modo analogo alla constatazione che la proprietà della casa non risolve la complessità del tema dell'abitare (Filandri, Semi, 2020), che comprende anche dimensioni occupazionali ed economiche e interagisce fortemente con il tema del welfare (Cellamare, 2020), così non è sufficiente risiedere in un territorio per abitarlo in modo congruente con i "funzionamenti di cittadinanza". La traduzione dai beni/risorse (tra cui la casa) in funzionamenti di cittadinanza dipende dalla capabilities (Sen, 1992). In questa accezione, la cittadinanza rimanda alla capacità pratica degli individui di realizzare combinazioni variabili di "funzionamenti" elementari (godere di buona salute, nutrirsi adeguatamente, abitare) e complessi (prendere parte alla vita della comunità, aumentare la propria autostima e progettualità). L'approccio delle capabilities richiede di considerare la gamma di possibilità/opportunità pratiche tra cui poi scegliere liberamente un funzionamento specifico (Bellanca, Biggeri, 2010). In questa prospettiva, la cittadinanza non è qualcosa che qualcuno ha (un set di diritti formali, tra cui la residenza), ma come qualcosa che qualcuno fa (Barbera, Negri, Salento, 2018). La cittadinanza è una forma di agire pratico, un'opportunità quotidiana fortemente dipendente dalle caratteristiche dei contesti. La "giusta distanza" costituisce una di queste caratte-

In apertura

"The New Wild", Alto Friuli, www.christopher thomson.net

(foto @) Christopher Thomson) ristiche e si declina a diversi livelli di scala, in tensione tra distinte polarità e in rapporto a specifici asset materiali e simbolici. Ciò che costituisce la giusta distanza per abitare da cittadino un territorio alpino, per esempio, non si ritrova in modo meccanico in un contesto urbano. Qualche indi- cazione per cogliere questa differenza si coglie già dai noti dati che caratterizzano il ruolo delle Terre Alte in Italia. A fronte del 58\% del territorio, la popolazione italiana che risiede nella Terre Alte è pari a circa un quarto del totale; il $76 \%$ del totale dei comuni appartiene alla c.d. classe dimensionale dei "comuni minimi" (meno di 2.000 abitanti), rispetto ad un'incidenza nazionale media del $60 \%$. Le Terre Alte raggruppano aree piuttosto diversificate, ma accomunate da vincoli e opportunità in gran parte simili: distanti dai grandi centri di agglomerazione e dai servizi, con strutture produttive rarefatte e fragilità demografiche, ma anche esempi di policentrismo, diversità, e con un forte potenziale per la produzione di servizi ecosistemici, cibo, paesaggi, cultura, energie rinnovabili e innovazione sociale. Quale è la "giusta distanza" per riabitare le Terre Alte?

\section{La "giusta distanza" come tratto dell'abitare metro-montano}

Irriducibilmente altra rispetto agli immaginari romantici che la volevano isolata, remota, separata fisicamente dal mondo moderno delle città e del mutamento sociale, la montagna alpina ha costruito negli ultimi sette secoli una civilizzazione complessa intorno all'asse pluridimensionale che possiamo definire della "giusta distanza". Un sistema di proporzioni, di spazi vuoti e pieni, di giustapposizioni che ritroviamo tanto sulla scala più ridotta che caratterizza il costruito e le modalità insediative tradizionali delle diverse forme dell'abitare alpino (dalla struttura dei borghi tipici delle Alpi latine, alle unità di vita e lavoro sparse sul territorio, esemplificati dai masi nelle Alpi germaniche), quanto sulla scala più ampia che storicamente ha inquadrato le relazioni socio-spaziali intercorrenti fra montagna e città (in termini di scambi economici e culturali, di spostamenti circolari di persone, fino al rispecchiarsi reciproco, l'una nella rappresentazione dell'altra, nelle epoche più recenti della nascita del turismo) (Zanzi, 2004; Mathieu, 1998).

Negli anni del secondo dopoguerra - perlomeno nei territori che hanno conosciuto il turismo di massa, la patrimonializzazione del paesaggio cul- 
turale e dei suoi manufatti e la sudditanza culturale ed economica alla città e ai suoi stili di vita - la distanza è andata diminuendo drasticamente, sino a collassare in tanti casi. Quella tra mondo montano e mondo urbano, innanzitutto, a causa di un avvicinarsi culturale (e spesso fisico, grazie ad una infrastrutturazione dei territori funzionale in primis al loro sfruttamento da parte della città, in termini anzitutto di velocità degli spostamenti) tra sistemi sino ad allora in relazione dialettica, laddove è la montagna del tracollo demografico e della profonda crisi dei valori tradizionali ad aver accettato di coincidere di fatto con l'orizzonte spaziale e simbolico dei poli urbani. E persino la distanza interna ai sistemi insediativi storici, come è evidente nei villaggi Walser, in cui lo spazio (di coltivazione e uso agricolo ma anche di "rispetto" comunitario) volutamente lasciato tra singoli edifici e tra i loro

Figg. 1-4

"The New Wild", Alto Friuli,

www.christopher thomson.net

(foto @ Christopher

Thomson). giusta distanza", che chiama in causa la necessità di ripensare l'infrastrutturazione del Paese uscendo dal dibattito "piccole/grandi opere".

Il movimento dei "nuovi montanari" (Corrado, Dematteis, Di Gioia, 2014) negli ultimi vent'anni, ha rappresentato la principale novità rispetto ad una ri-tematizzazione della "giusta distanza" in termini contemporanei nel contesto alpino. I giovani (e non solo), spesso altamente qualificati e fortemente motivati, che hanno lasciato le aree metropolitane per andare a vivere e a lavorare in montagna (Barbera, Dagnes, Membretti, 2018), esprimono una tensione concreta verso nuove modalità di riabitare le Alpi (quelle meno turisticizzate, più interne, ancorché non tagliate fuori dalle relazioni col mondo di pianura): modalità centrate sulla ricerca di un diverso equilibrio (spaziale e culturale) tra mondi non più così distanti ma che richiedono appunto nuove forme di interazione, nuovi spazi "tra" (in-between), che garantiscano il reciproco riconoscimento tra le parti. Possiamo affermare che, prima della rivoluzione spazialista innescata dalla pandemia del Covid-19, proprio in queste pratiche e in queste concezioni del territo-

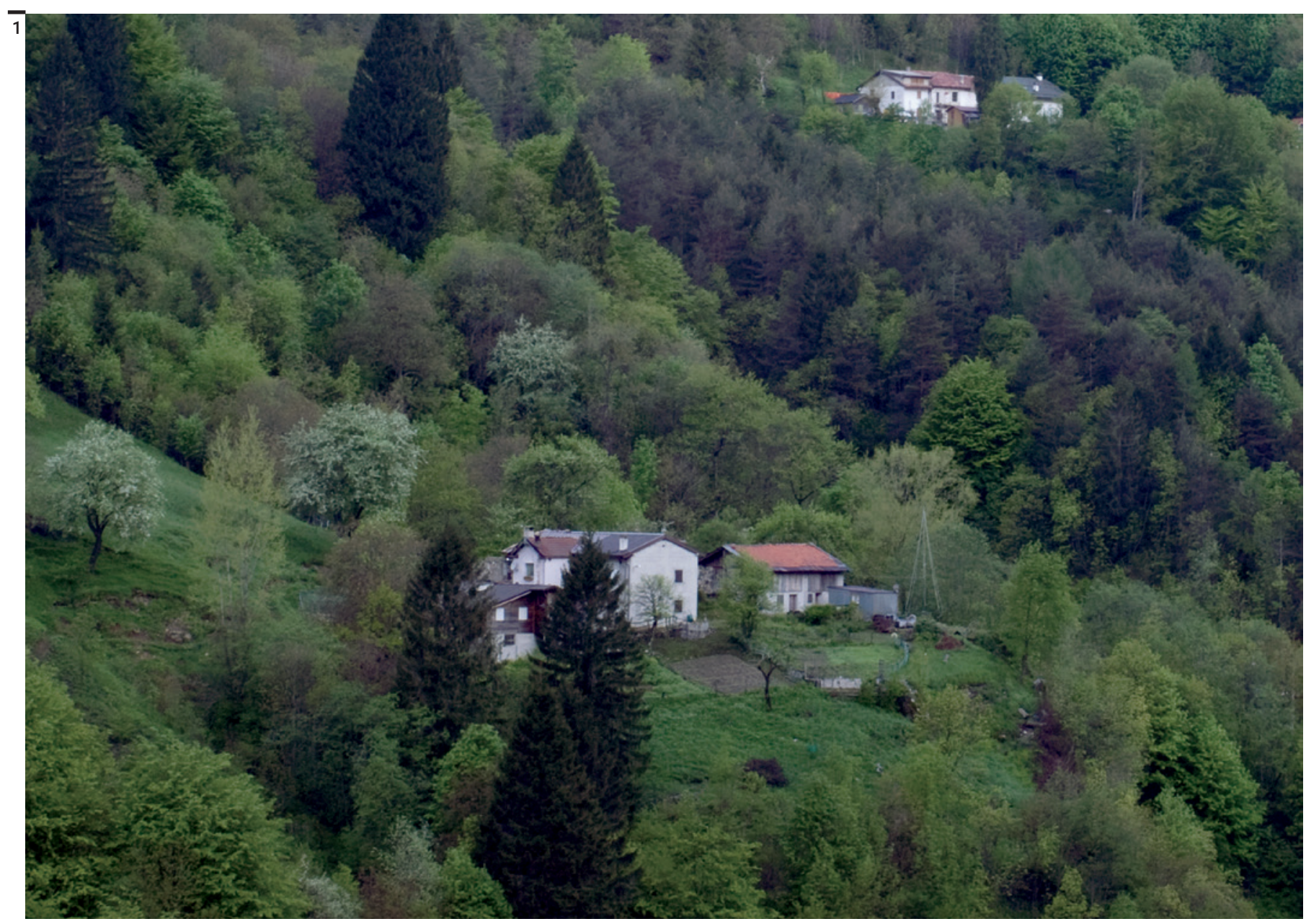



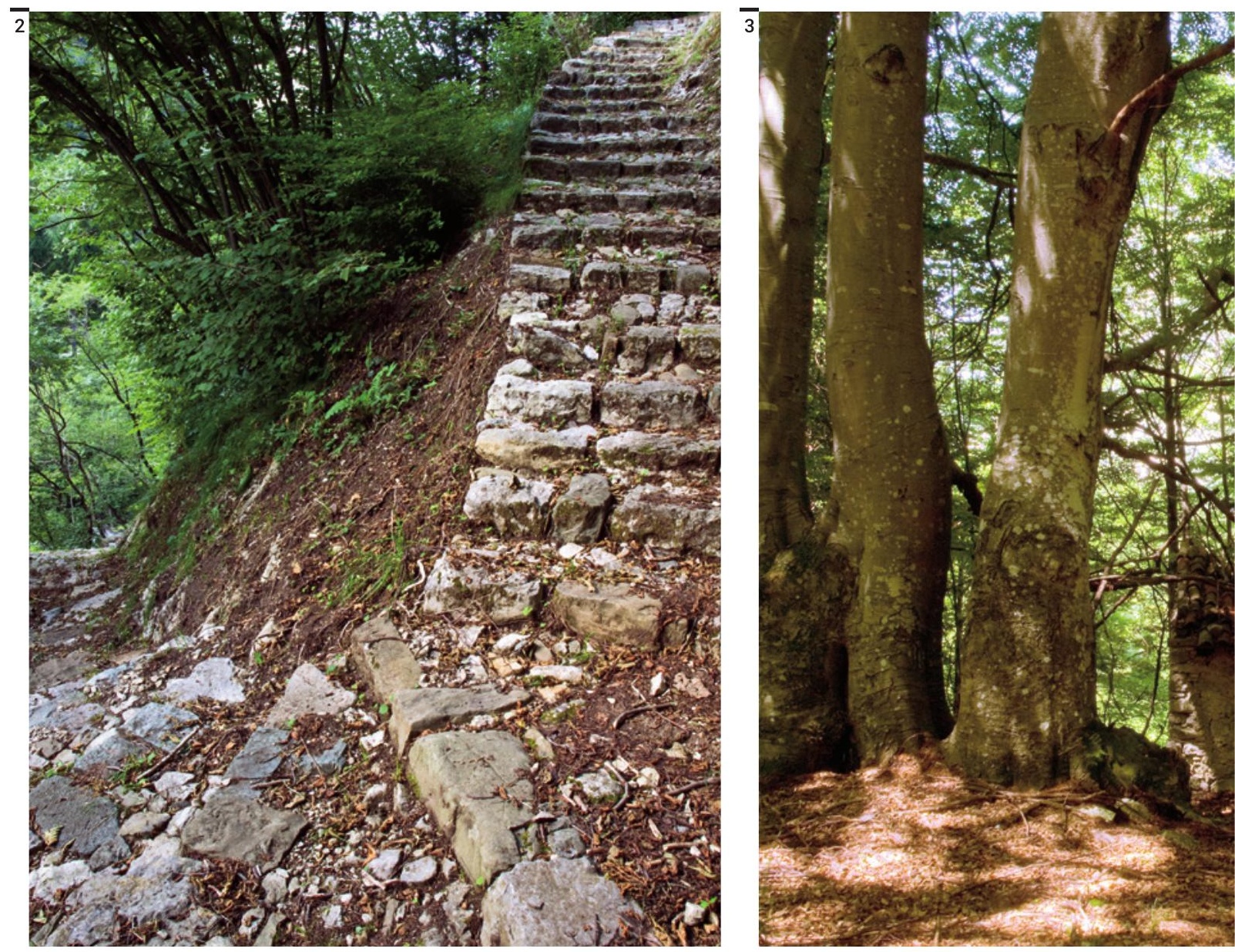

rio si manifesta l'idea di un necessario (ri)distanziamento, fisico e sociale, di una forma di dispersione abitativa e lavorativa, ancorché dentro le dinamiche e i flussi della globalizzazione.

I "nuovi montanari", per quanto fenomeno quantitativamente modesto, hanno contribuito dunque in modo sostanziale (insieme a quel filone artistico e culturale sulla montagna che si è sviluppato negli ultimi anni, di cui l'opera e la figura dello scrittore Paolo Cognetti rappresenta un caso emblematico) a reinventare in una certa misura le due polarità tra cui la distanza (oggi spesso più simbolica che fisica) si dispiega: ovvero a vederle come parte di un tutto - che possiamo chiamare tecnicamente il sistema metro-montano - Barbera, 2020 https://www.che-fare.com/barbera-innovazione-metromontana-citta-metropolitana/) in cui lo spazio che separa (città e montagna, così come un insediamento alpino dall'altro) è generatore di senso, è categoria della conoscenza, o più semplicemente rappresenta il quotidiano elemento che consente la relazione tra il qui e l'altrove. In questo distanziamento, non ancora imposto per legge dalle misure emergenziali anti pandemiche, la rarefazione socio-spaziale che caratterizza oggi tan- ta parte delle Terre Alte può allora tramutarsi in risorsa per l'innovazione - il "vuoto creativo" di cui parla Francesco Remotti - (Remotti, 2011), in occasione per sperimentare quotidianamente "un più largo respiro" esistenziale e di vita quotidiana, per citare Luisa Bonesio e il suo approccio al paesaggio abitato (Bonesio, 2017).

La pandemia del Covid-19, con il correlato di norme per il distanziamento sociale e l'immobilità residenziale, sembra aver definito un quadro nuovo e nuove potenzialità rispetto a questo fenomeno, in particolare per quanto riguarda le aree montane. Improvvisamente ci siamo tutti trovati a ripensare radicalmente il nostro essere nel mondo, o meglio il nostro stare: sono state messe in radicale discussione due spinte parallele, quella alla mobilità e quella alla prossimità - definite da John Urry come compulsion to mobility, compulsion to proximity (Urry, 2002), su cui sino a ieri si è basato il sistema socio-spaziale contemporaneo.

Di fronte alla prospettiva di trascorrere a casa, o comunque in porzioni di territorio limitate, periodi significativi della nostra esistenza (perlomeno periodicamente), le grandi città non sembrano più essere i luoghi più desiderabili per vivere. I gran- 

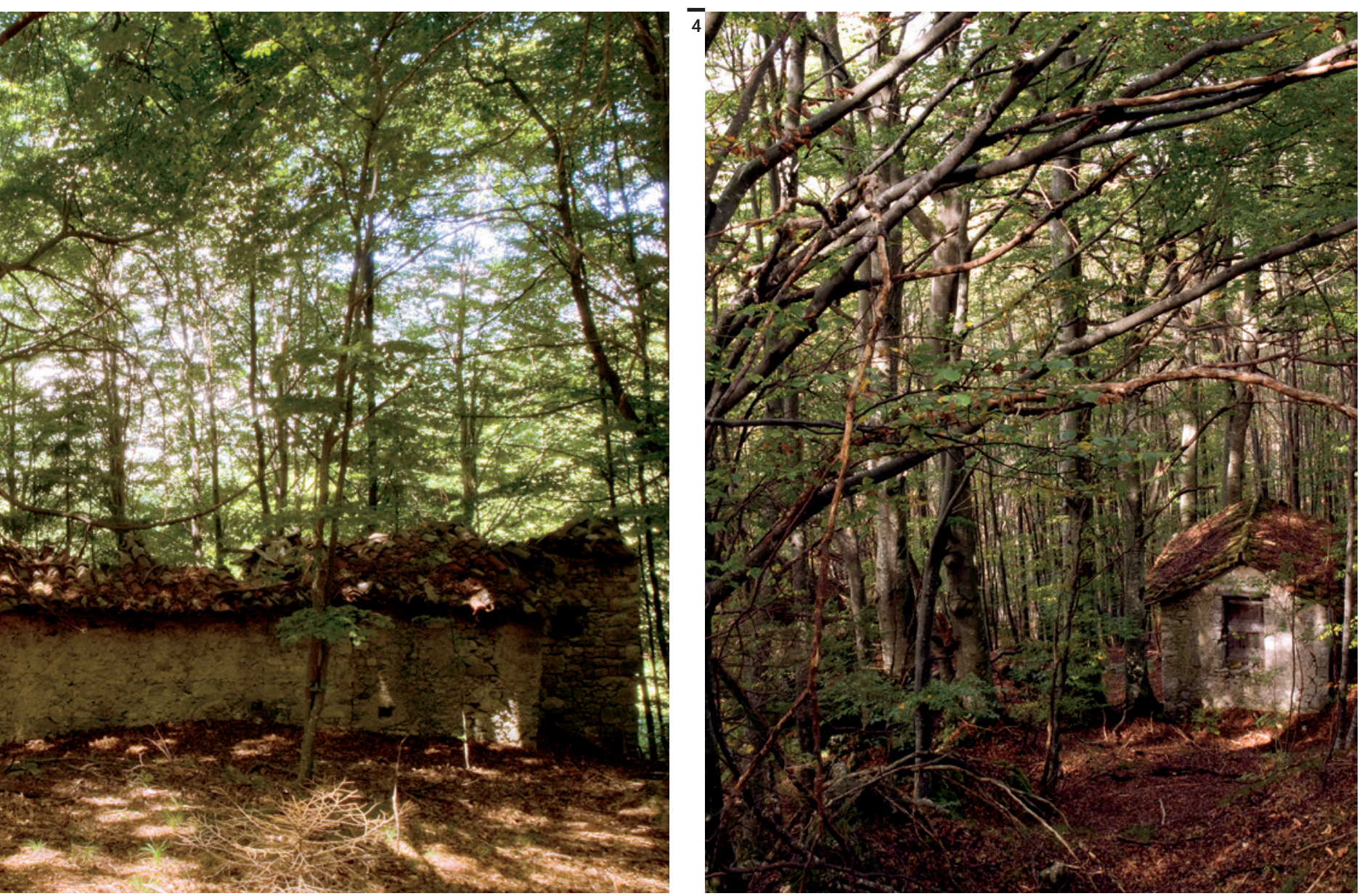

di numeri di persone, la prossimità forzosa con una folla anonima potenzialmente fonte di rischio, gli attraversamenti del territorio regolati da norme iper securitarie, la mancanza di spazi aperti, di natura fuori della porta di casa, che possa compensare la minore possibilità di viaggiare: sono alcuni dei fattori che potrebbero spingere una parte della popolazione, perlomeno quella con le risorse culturali ed economiche necessarie, a cercare la "giusta distanza", trasferendosi (anche in modo intermittente) verso aree meno densamente popolate, come quelle interne e montane. Questo naturalmente a fronte della possibilità di connessione a Internet (che consentano lo smart working), di una infrastrutturazione di base efficiente (dal negozio di alimentari in paese alla strada di collegamento con la città tenuta in buone condizioni, ai servizi di base decentrati, quali quelli per l'infanzia o per la salute) e naturalmente di possibilità lavorative concrete (sia di lavoro a distanza, sia in loco, con la riscoperta di economie a Km zero, di filiere corte). Il movimento dei "nuovi montanari" sembra allora configurarsi come una possibile avanguardia, apripista rispetto a nuove tendenze sociali (nelle quali immaginari e necessità tendono a mescolarsi in modi inediti), la cui portata futura è tuttavia ancora tutta da definire. Si apre forse una nuova stagione nelle Alpi per inventare nuove politiche dei luoghi, per immaginare e per sostenere pratiche di ri-territorializzazione, per favorire non l'isolamento dal mondo ma una diversa forma di (inter)connessione, su scala diversa, tra locale e globale, tra città e montagna?

Una stagione in cui il distanziamento sociale venga trasformato da obbligo a scelta, e con esso un passaggio a nuove forma di stanzialità, di radicamento locale, una sorta di compulsion to locality (Membretti, 2020) in contrasto con la precedente compulsione alla iper mobilità, al nomadismo post moderno.

Come racconta l'antropologa Maria Molinari nel volume che ha recentemente pubblicato sul piccolo borgo appenninico di Berceto, ci sono dei vantaggi nel vivere "un po' in disparte", nel "guardare al mondo lateralmente" (Molinari, 2020): specialmente se è il mondo post Covid-19.

Uno sguardo, quello dei neo abitanti e dei "ritornanti" nelle terre alte, che si basa sulla lentezza, sulle reti corte di vicinato, sulla prossimità data dal condividere la cura di un territorio vissuto come 


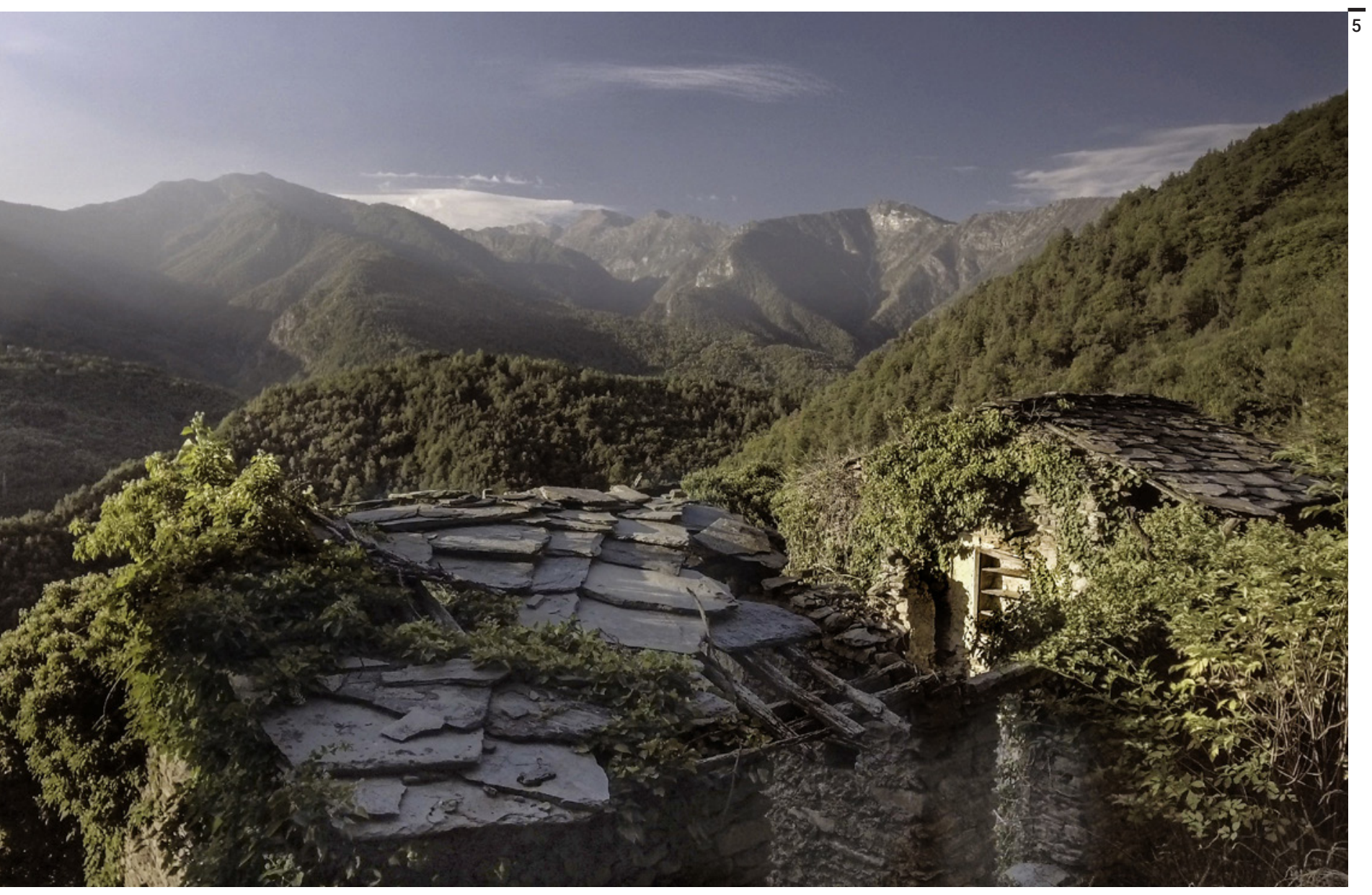

Fig. 5 bene comune. Ma, nel contempo, uno sguardo La casa del sole che mette in relazione il qui e l'altrove, che non (foto Paolo Maitre si accontenta di rivolgersi solo al proprio cortiLibertini). le, cercando piuttosto di leggere le trasformazioni del mondo con le lenti della dimensione locale, in

Fig. 6 un'ottica trans locale.

Bourcet (foto Paolo Maitre Libertini).

\section{Conclusioni. Luoghi (e) immaginari}

La ricerca della "giusta distanza" emerge in piena pandemia come risposta immediata nel calo della ricerca della casa e di "metrofilia" residenziale. Uno studio pubblicato da una nota società immobiliare, evidenzia che nel periodo pre-pandemia circa il 34\% delle ricerche di abitazioni a livello nazionale interessavano capoluoghi di provincia, mentre da quando sono in atto le misure restrittive la ricerca si è abbassata di vari punti percentuali a favore dei piccoli centri. Questa "giusta distanza" nelle preferenze di ricerca si accompagna a una "giusta distanza" simbolica. Nei luoghi della "restanza", come la chiama Vito Teti (Teti, 2014) e del ritorno (o del neo popolamento) si apre uno scenario inedito: quello della ricerca, per forza e per scelta, di una "giusta distanza" definita da comunità di destino, che intendono reagire alla delocalizzazione, alla periferizzazione imposta da centri economico-finanziari e dalle grandi aree metropolitane, all'insicurezza fisica e sociale prodotta dagli spazi della concentrazione, della densità. Quei fenomeni di accentramento ad alto impatto ambientale ed umano che si sono affermati con prepotenza sul tessuto dell'abitare diffuso, quello che era sino a pochi decenni fa la base dell'antropizzazione, del lavoro e del vivere in un paese di origine rurale come l'Italia. Una "giusta distanza" che può essere antidoto alla fuga disorganizzata e individuale di fronte al virus, per ceti benestanti che se lo possono permettere, laddove riabitare i luoghi marginalizzati (come ci racconta il progetto collettivo dell'associazione "Riabitare l'Italia") è invece il frutto di una inversione politica dello sguardo, di un mutamento radicale e collettivo di prospettiva, che forse proprio la pandemia va sollecitando. 


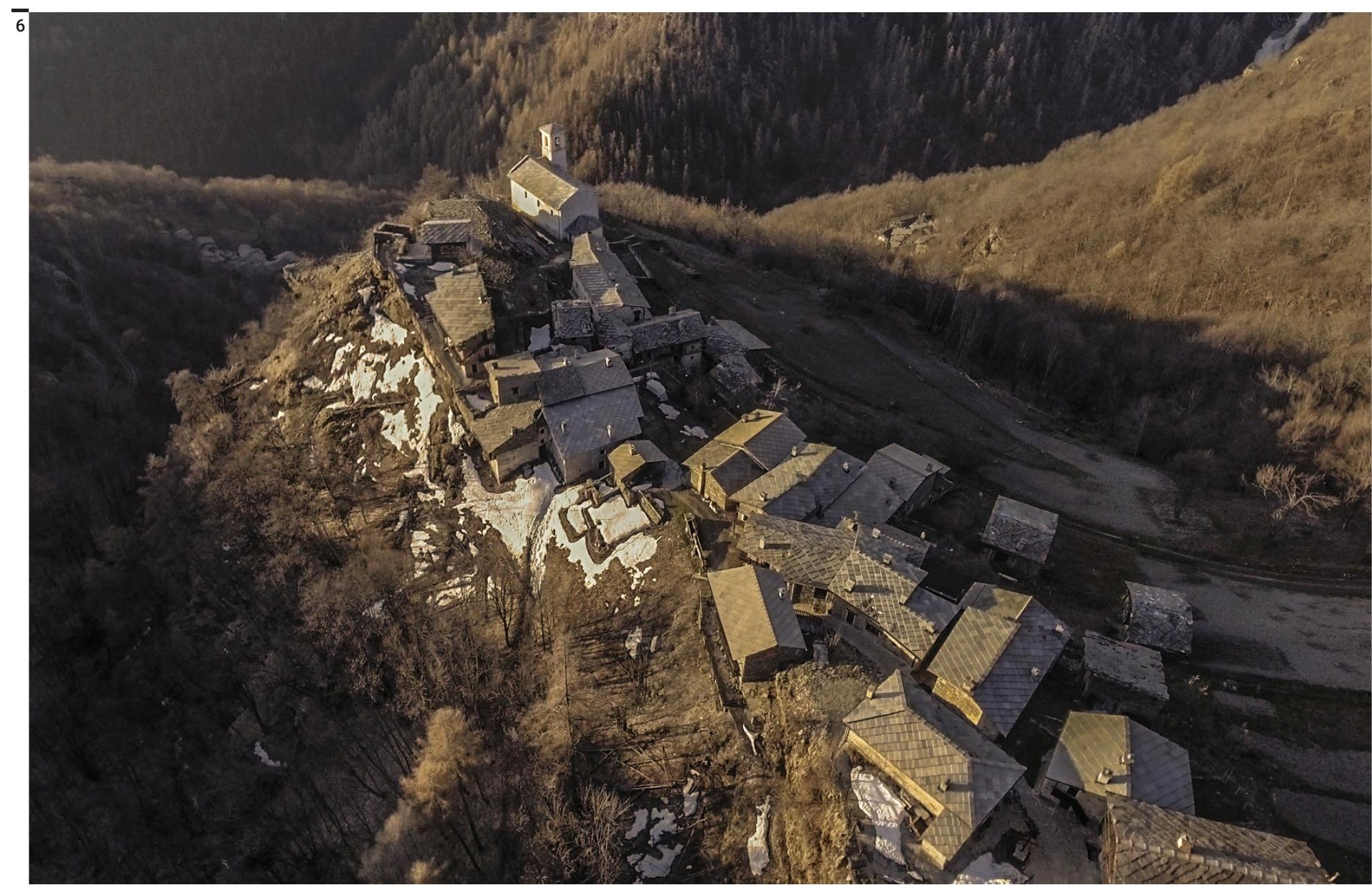

\section{Bibliografia}

Barbera Filippo, Negri Nicola, Salento Angelo (2018), «From individual choice to collective voice: foundational economy, local commons and citizenship», in Rassegna italiana di Sociologia, a. LVIX, n. 2, aprile-giugno, pp. 371-397. Bellanca Nicolò, Biggeri Mario (a cura di) (2010), Dalla relazione di cura alla relazione di prossimità, Liguori, Napoli. Bonesio Luisa (2017), «Un più largo respiro. Rappresentazioni dell'aria nella modernità», in Dei Cas Livio, Schena Leo, Memorie dell'Aria in Alta Valle, Cssav, Sondrio.

Corrado Federica, Dematteis Giuseppe, Di Gioia Alberto (2014), Nuovi montanari. Abitare le Alpi nel XXI secolo, Franco Angeli, Milano.

Cellamare Carlo, Cognetti Francesca, Guidarini Stefano, Marchigiani Elena (2020), «Edilizia residenziale pubblica: un campo strategico tra case e città», relazione presentata al convegno Ricomporre i divari. Progetti e politiche territoriali contro le disuguaglianze, 17-18 febbraio 2020, Politecnico di Milano, DAStU-Dipartimento d'Eccellenza sulle Fragilità territoriali 2018-2022 in collaborazione col Forum Disuguaglianze Diversità.

Filandri Marianna, Semi Giovanni (2020), «Proprietà e domanda sociale di casa», relazione presentata al convegno Ricomporre i divari. Progetti e politiche territoriali contro le disuguaglianze, 17-18 febbraio 2020, Politecnico di Milano, DAStU-Dipartimento d'Eccellenza sulle Fragilità territoriali 2018-2022 in collaborazione col Forum Disuguaglianze Diversità.

Mathieu Jon (1998), Geschichte der Alpen 1500-1900. Umwelt, Entwicklung, Gesellschaft, Böhlau, Wien.

Membretti Andrea (2020), «Compulsion to Locality? Mobility, proximity and the role of rural and mountain areas after the Covid-19 crisis», abstract presentato alla conferenza internazionale Bodies in the climate change era, University of Konkuk (South Korea), 29-05-2020. https://www.researchgate.net/publication/341110633_ Membretti_Compulsion_to_Locality_Preprint.

Molinari Maria (2020), Un territorio immaginato. Vecchie e nuove immigrazioni in un paese dell'Appennino, MUP, Parma.

Remotti Francesco (2011), «Impoverimento e creatività», in Id., Cultura. Dalla complessità all'impoverimento, Laterza, Roma-Bari, pp. 281-301.

Sen Amartya Kumar (1992), Inequality Reexamined, Oxford University Press, Oxford.

Teti Vito (2014), Pietre di pane. Un'antropologia del restare, Quodlibet, Macerata.

Urry John (2002), «Mobility and Proximity», in Sociology Journal, vol. 36 n. 2, pp. 255-274.

Zanzi Luigi (2004), Le Alpi nella storia d'Europa, Cda \& Vivalda, Torino. 